\title{
Research Article \\ Further Development of Jarratt Method for Solving Nonlinear Equations
}

\author{
R. Thukral \\ Padé Research Centre, 39 Deanswood Hill, Leeds, West Yorkshire LS17 5JS, UK \\ Correspondence should be addressed to R. Thukral, rthukral@hotmail.co.uk
}

Received 18 May 2012; Accepted 17 July 2012

Academic Editor: Michele Benzi

Copyright (C) 2012 R. Thukral. This is an open access article distributed under the Creative Commons Attribution License, which permits unrestricted use, distribution, and reproduction in any medium, provided the original work is properly cited.

We present two new families of Jarratt-type methods for solving nonlinear equations. It is proved that the order of convergence of each family member is improved from four to six by the addition of one function evaluation. Per iteration, these new methods require two evaluations of the function and two evaluations of the first-order derivatives. In fact, the efficiency index of these methods is 1.565. Numerical comparisons are made with other existing methods to show the performance of the presented methods.

\section{Introduction}

Multipoint iterative methods for solving nonlinear equations are of great practical importance since they overcome theoretical limits of one-point methods concerning the convergence order and computational efficiency. The new iterative methods are applied to find a simple root $\alpha$ of the nonlinear equation

$$
f(x)=0,
$$

where $f: D \subset \mathbb{R} \rightarrow \mathbb{R}$ is a scalar function on an open interval $D$, and it is sufficiently smooth in a neighbourhood of $\alpha$. In this paper, we present new variants of the Jarratt method, based on the composition of Jarratt and Newton-type methods instead of a twostep Newton method. The new variants are formed by adding an evaluation of the function at another point in the procedure iterated by the Jarratt method. Consequently, the order of convergence of the new Jarratt-type method is improved from four to six. Per iteration, the new methods require two evaluations of the function and two of its first derivatives. Recently, many variants of the Jarratt method with sixth-order convergence have been developed [1$3]$, which improve the order of convergence of the Jarratt method by an additional evaluation 
of the function. Hence, the prime motive of this study is to develop a new class of efficient Jarratt-type methods for solving nonlinear equations. In addition, these new sixth-order Jarratt-type methods have an efficiency index equivalent to the recently established methods presented in [1-3]. Consequently, we have found that the new Jarratt-type methods are efficient and robust.

The paper is organized as follows. In Section 2, two new families of the Jarratttype method of sixth-order are developed. Convergence analysis is provided to establish their sixth order of convergence. In Section 3, we briefly state the established methods in order to compare the effectiveness of the new Jarratt-type methods. Finally, in Section 4, several numerical examples are given to demonstrate the performance of the new Jarratt-type methods presented in this contribution.

\section{Development of the Methods and Convergence Analysis}

In this section we define two new sixth-order Jarratt-type methods. The new scheme was actually developed by using the classical Jarratt method and then squaring the weight function in the third step. We begin with the classical Jarratt method and then show the improvement in the third step.

Let

$$
J\left(x_{n}\right)=\frac{3 f^{\prime}\left(y_{n}\right)+f^{\prime}\left(x_{n}\right)}{6 f^{\prime}\left(y_{n}\right)-2 f^{\prime}\left(x_{n}\right)}
$$

then a new improved Jarratt-type method is given as

$$
\begin{gathered}
y_{n}=x_{n}-\left(\frac{2}{3}\right)\left(\frac{f\left(x_{n}\right)}{f^{\prime}\left(x_{n}\right)}\right), \\
z_{n}=x_{n}-J\left(x_{n}\right)\left(\frac{f\left(x_{n}\right)}{f^{\prime}\left(x_{n}\right)}\right), \\
x_{n+1}=z_{n}-J\left(x_{n}\right)^{2}\left(\frac{f\left(z_{n}\right)}{f^{\prime}\left(x_{n}\right)}\right) .
\end{gathered}
$$

We observe that the new Jarratt-type method (2.2) is simply formed to attain the sixth order of convergence. Using this concept we developed three different families of sixth-order methods.

In order to establish the order of convergence of these new Jarratt-type methods we state the three essential definitions.

Definition 2.1. Let $f(x)$ be a real function with a simple root $\alpha$, and let $\left\{x_{n}\right\}$ be a sequence of real numbers that converge towards $\alpha$. The order of convergence $m$ is given by

$$
\lim _{n \rightarrow \infty} \frac{x_{n+1}-\alpha}{\left(x_{n}-\alpha\right)^{m}}=\rho \neq 0
$$

where $\rho$ is the asymptotic error constant and $m \in \mathbb{R}^{+}$. 
Definition 2.2. Let $\lambda$ be the number of function evaluations of the new method. The efficiency of the new method is measured by the concept of efficiency index $[4,5]$ and defined as

$$
\mu^{1 / \lambda}
$$

where $\mu$ is the order of the method.

Definition 2.3. Suppose that $x_{n-1}, x_{n}$, and $x_{n+1}$ are three successive iterations closer to the root $\alpha$ of (1.1). Then the computational order of convergence [6] may be approximated by

$$
\mathrm{COC} \approx \frac{\ln \left|\left(x_{n+1}-\alpha\right)\left(x_{n}-\alpha\right)^{-1}\right|}{\ln \left|\left(x_{n}-\alpha\right)\left(x_{n-1}-\alpha\right)^{-1}\right|}
$$

where $n \in \mathbb{N}$.

\subsection{The Three-Point Jarratt-Type Methods}

We consider the following iteration scheme:

$$
\begin{gathered}
y_{n}=x_{n}-\left(\frac{2}{3}\right)\left(\frac{f\left(x_{n}\right)}{f^{\prime}\left(x_{n}\right)}\right), \\
z_{n}=x_{n}-\left(\frac{3 f^{\prime}\left(y_{n}\right)+f^{\prime}\left(x_{n}\right)}{6 f^{\prime}\left(y_{n}\right)-2 f^{\prime}\left(x_{n}\right)}\right)\left(\frac{f\left(x_{n}\right)}{f^{\prime}\left(x_{n}\right)}\right), \\
x_{n+1}=z_{n}-\left(\frac{\beta f^{\prime}\left(y_{n}\right)+(4-\beta) f^{\prime}\left(x_{n}\right)}{(\beta+3) f^{\prime}\left(y_{n}\right)+(1-\beta) f^{\prime}\left(x_{n}\right)}\right)^{2}\left(\frac{f\left(z_{n}\right)}{f^{\prime}\left(x_{n}\right)}\right) .
\end{gathered}
$$

The first two steps of (2.6) are the same as those of the classical fourth-order Jarratt method [7], and the third step is constructed to achieve the sixth order of convergence. In order to achieve the sixth order of convergence, we introduce a weight function in the third step of (2.6). To obtain the solution of (1.1) by the new sixth-order Jarratt-type methods, we must set a particular initial approximation $x_{0}$, ideally close to the simple root and provided that the denominators in (2.6) are not equal to zero. In numerical mathematics it is essential to know the behaviour of an approximate method. Therefore, we prove the order of convergence of the new sixth-order methods.

Theorem 2.4. Assume that the function $f: D \subset \mathbb{R} \rightarrow \mathbb{R}$ for an open interval $D$ has a simple root $\alpha \in D$. If $f(x)$ is sufficiently smooth in the neighbourhood of the root $\alpha$, then the method defined by (2.6) is of order six.

Proof. Let $\alpha$ be a simple root of $f(x)$, that is, $f(\alpha)=0$ and $f^{\prime}(\alpha) \neq 0$, and the error is expressed as

$$
e=x-\alpha .
$$


Using Taylor expansion, we have

$$
f\left(x_{n}\right)=f(\alpha)+f^{\prime}(\alpha) e_{n}+2^{-1} f^{\prime \prime}(\alpha) e_{n}^{2}+6^{-1} f^{\prime \prime \prime}(\alpha) e_{n}^{3}+24^{-1} f^{i v}(\alpha) e_{n}^{4}+\cdots
$$

Taking $f(\alpha)=0$ and simplifying, expression (2.8) becomes

$$
f\left(x_{n}\right)=f^{\prime}(\alpha)\left[e_{n}+c_{2} e_{n}^{2}+c_{3} e_{n}^{3}+c_{4} e_{n}^{4}+\cdots\right],
$$

where $n \in \mathbb{N}$ and

$$
c_{k}=\frac{f^{(k)}(\alpha)}{(k !) f^{\prime}(\alpha)} \quad \text { for } k \geq 2
$$

Furthermore, we have

$$
f^{\prime}\left(x_{n}\right)=f^{\prime}(\alpha)\left[1+2 c_{2} e_{n}+3 c_{3} e_{n}^{2}+4 c_{4} e_{n}^{3}+\cdots\right] .
$$

Dividing (2.9) by (2.11), we get

$$
\frac{f\left(x_{n}\right)}{f^{\prime}\left(x_{n}\right)}=e_{n}-c_{2} e_{n}^{2}+2\left(c_{2}^{2}-c_{3}\right) e_{n}^{3}+\left(7 c_{2} c_{3}-4 c_{2}^{3}-3 c_{4}\right) e_{n}^{4}+\cdots,
$$

and, hence, we have

$$
y_{n}-\alpha=e_{n}-\frac{2}{3} \frac{f\left(x_{n}\right)}{f^{\prime}\left(x_{n}\right)}=\frac{1}{3} e_{n}+\frac{2}{3}\left[c_{2} e_{n}^{2}-2\left(c_{2}^{2}-c_{3}\right) e_{n}^{3}-\left(7 c_{2} c_{3}-4 c_{2}^{3}-3 c_{4}\right) e_{n}^{4}\right]+\cdots
$$

The expansion of $f^{\prime}\left(y_{n}\right)$ about $\alpha$ is given as

$$
f^{\prime}\left(y_{n}\right)=f^{\prime}(\alpha)\left[1+\frac{2}{3} c_{2} e_{n}+\frac{1}{3}\left(4 c_{2}^{2}+c_{3}\right) e_{n}^{2}-\left(\frac{8}{3} c_{2}^{3}-4 c_{2} c_{3}-\frac{4}{27} c_{4}\right) e_{n}^{3}+\cdots\right] \text {. }
$$

From (2.11) and (2.14) we attain

$$
\begin{gathered}
3 f^{\prime}\left(y_{n}\right)+f^{\prime}\left(x_{n}\right)=4 f^{\prime}(\alpha)\left[1+c_{2} e_{n}+\left(c_{2}^{2}+c_{3}\right) e_{n}^{2}+\left(3 c_{2} c_{3}-2 c_{2}^{3}+\frac{10}{9} c_{4}\right) e_{n}^{3}+\cdots\right] \\
6 f^{\prime}\left(y_{n}\right)-2 f^{\prime}\left(x_{n}\right)=4 f^{\prime}(\alpha)\left[1+\left(2 c_{2}^{2}-c_{3}\right) e_{n}^{2}+\left(6 c_{2} c_{3}-4 c_{2}^{3}-\frac{16}{9} c_{4}\right) e_{n}^{3}+\cdots\right] .
\end{gathered}
$$

Dividing (2.15) by (2.16) gives us

$$
J\left(x_{n}\right)=\frac{3 f^{\prime}\left(y_{n}\right)+f^{\prime}\left(x_{n}\right)}{6 f^{\prime}\left(y_{n}\right)-2 f^{\prime}\left(x_{n}\right)}=1+c_{2} e_{n}-\left(c_{2}^{2}-2 c_{3}\right) e_{n}^{2}-2\left(c_{2} c_{3}-\frac{13}{9} c_{4}\right) e_{n}^{3}+\cdots,
$$


From (2.12) and (2.17), we have

$$
J\left(x_{n}\right) \frac{f\left(x_{n}\right)}{f^{\prime}\left(x_{n}\right)}=e_{n}-\left(c_{2}^{3}-c_{2} c_{3}+\frac{1}{9} c_{4}\right) e_{n}^{4}+\cdots
$$

Thus from (2.6) and (2.18), we have

$$
e_{n}-\alpha=e_{n}-J\left(x_{n}\right) \frac{f\left(x_{n}\right)}{f^{\prime}\left(x_{n}\right)}=\left(c_{2}^{3}-c_{2} c_{3}+\frac{1}{9} c_{4}\right) e_{n}^{4}+\cdots
$$

For the third step, we expand $f\left(z_{n}\right)$ about $\alpha$, and we have

$$
f\left(z_{n}\right)=f^{\prime}(\alpha)\left[\left(c_{2}^{3}-c_{2} c_{3}+\frac{1}{9} c_{4}\right) e_{n}^{4}+\cdots\right]
$$

The expansion of the weight function used in the third step (2.6) is given as

$$
\left[\frac{\beta f^{\prime}\left(y_{n}\right)+(4-\beta) f^{\prime}\left(x_{n}\right)}{(\beta+3) f^{\prime}\left(y_{n}\right)+(1-\beta) f^{\prime}\left(x_{n}\right)}\right]^{2}=1+2 c_{2} e_{n}+3^{-1}\left(12 c_{3}+2 \beta c_{2}^{2}-9 c_{2}^{2}\right) e_{n}^{2}+\cdots
$$

Substituting appropriate expressions in the third step of (2.6), we obtain

$$
e_{n+1}=-3^{-3}\left(c_{4}-9 c_{2} c_{3}+9 c_{2}^{3}\right)\left(3 c_{3}-9 c_{2}^{2}+2 \beta c_{2}^{2}\right) e_{n}^{6}+\cdots
$$

The expression (2.22) establishes the asymptotic error constant for the sixth order of convergence for the new Jarratt-type method defined by (2.6).

\subsection{Method 2}

The second of the new three-step Jarratt-type method is given as

$$
\begin{gathered}
y_{n}=x_{n}-\left(\frac{2}{3}\right)\left(\frac{f\left(x_{n}\right)}{f^{\prime}\left(x_{n}\right)}\right), \\
z_{n}=x_{n}-\left(\frac{3 f^{\prime}\left(y_{n}\right)+f^{\prime}\left(x_{n}\right)}{6 f^{\prime}\left(y_{n}\right)-2 f^{\prime}\left(x_{n}\right)}\right)\left(\frac{f\left(x_{n}\right)}{f^{\prime}\left(x_{n}\right)}\right), \\
x_{n+1}=z_{n}-\left(\frac{\beta f^{\prime}\left(y_{n}\right)^{2}+(4-\beta) f^{\prime}\left(x_{n}\right)^{2}}{(\beta+3) f^{\prime}\left(y_{n}\right)^{2}+(1-\beta) f^{\prime}\left(x_{n}\right)^{2}}\right)\left(\frac{f\left(z_{n}\right)}{f^{\prime}\left(x_{n}\right)}\right) .
\end{gathered}
$$

$x_{0}$ is the initial approximation and provided that the denominators of (2.23) are not equal to zero. 
Theorem 2.5. Assume that the function $f: D \subset \mathbb{R} \rightarrow \mathbb{R}$ for an open interval $D$ has a simple root $\alpha \in D$. If $f(x)$ is sufficiently smooth in the neighbourhood of the root $\alpha$, then the method defined by (2.23) is of order six.

Proof. Using appropriate expressions in the proof of the Theorem 2.4 and substituting them into (2.23), we obtain the asymptotic error constant

$$
e_{n+1}=-3^{-3}\left(c_{4}-9 c_{2} c_{3}+9 c_{2}^{3}\right)\left(3 c_{3}-10 c_{2}^{2}+4 \beta c_{2}^{2}\right) e_{n}^{6}+\cdots
$$

The expression (2.24) establishes the asymptotic error constant for the sixth order of convergence for the new Jarratt-type method defined by (2.23).

\subsection{Method 3}

The third of the new three-step Jarratt-type method is expressed as

$$
\begin{gathered}
y_{n}=x_{n}-\left(\frac{2}{3}\right)\left(\frac{f\left(x_{n}\right)}{f^{\prime}\left(x_{n}\right)}\right), \\
z_{n}=x_{n}-\left(\frac{3 f^{\prime}\left(y_{n}\right)+f^{\prime}\left(x_{n}\right)}{6 f^{\prime}\left(y_{n}\right)-2 f^{\prime}\left(x_{n}\right)}\right)\left(\frac{f\left(x_{n}\right)}{f^{\prime}\left(x_{n}\right)}\right), \\
x_{n+1}=z_{n}-\left(\frac{(2 \beta+1) f^{\prime}\left(y_{n}\right)^{2}+(2 \beta+3) f^{\prime}\left(x_{n}\right)^{2}}{(5 \beta+4) f^{\prime}\left(y_{n}\right)^{2}-\beta f^{\prime}\left(x_{n}\right)^{2}}\right)\left(\frac{f\left(z_{n}\right)}{f^{\prime}\left(x_{n}\right)}\right) .
\end{gathered}
$$

$x_{0}$ is the initial approximation and provided that the denominators of (2.25) are not equal to zero.

Theorem 2.6. Assume that the function $f: D \subset \mathbb{R} \rightarrow \mathbb{R}$ for an open interval $D$ has a simple root $\alpha \in D$. If $f(x)$ is sufficiently smooth in the neighbourhood of the root $\alpha$, then the method defined by (2.25) is of order six.

Proof. Using appropriate expressions in the proof of the Theorem 2.4 and substituting them into (2.25), we obtain the asymptotic error constant

$$
e_{n+1}=-3^{-3}(1-\beta)^{-1}\left(c_{4}-9 c_{2} c_{3}+9 c_{2}^{3}\right)\left(6 c_{2}^{2}-3 c_{3}+2 \beta c_{2}^{2}-3 \beta c_{3}\right) e_{n}^{6}+\cdots
$$

The expression (2.26) establishes the asymptotic error constant for the sixth order of convergence for the new Jarratt-type method defined by (2.25).

\section{The Established Jarratt-Type Sixth-Order Methods}

For the purpose of comparison, we consider three sixth-order methods presented recently in [1-3]. Since these methods are well established, we will state the essential expressions used in order to calculate the approximate solution of the given nonlinear equations and thus compare the effectiveness of the new sixth order Jarratt-type methods. 


\subsection{Wang et al. Method}

In [3], Wang et al. developed another three-step sixth order of convergence method, and the particular expressions of the method are given as

$$
\begin{gathered}
y_{n}=x_{n}-\left(\frac{2}{3}\right)\left(\frac{f\left(x_{n}\right)}{f^{\prime}\left(x_{n}\right)}\right), \\
z_{n}=x_{n}-\left(\frac{3 f^{\prime}\left(y_{n}\right)+f^{\prime}\left(x_{n}\right)}{6 f^{\prime}\left(y_{n}\right)-2 f^{\prime}\left(x_{n}\right)}\right)\left(\frac{f\left(x_{n}\right)}{f^{\prime}\left(x_{n}\right)}\right), \\
x_{n+1}=z_{n}-\left(\frac{f\left(z_{n}\right)}{2}\right)\left(\frac{3}{f^{\prime}\left(y_{n}\right)}-\frac{1}{f^{\prime}\left(x_{n}\right)}\right) .
\end{gathered}
$$

\subsection{Wang et al. Method}

In [2], Wang et al. developed a three-step sixth order of convergence method, as before we state the essential expressions used in the method as follows:

$$
\begin{gathered}
y_{n}=x_{n}-\left(\frac{2}{3}\right)\left(\frac{f\left(x_{n}\right)}{f^{\prime}\left(x_{n}\right)}\right), \\
z_{n}=x_{n}-\left(\frac{3 f^{\prime}\left(y_{n}\right)+f^{\prime}\left(x_{n}\right)}{6 f^{\prime}\left(y_{n}\right)-2 f^{\prime}\left(x_{n}\right)}\right)\left(\frac{f\left(x_{n}\right)}{f^{\prime}\left(x_{n}\right)}\right), \\
x_{n+1}=z_{n}-\left(\frac{(5 \mu+\beta) f^{\prime}\left(y_{n}\right)-(3 \mu+\beta) f^{\prime}\left(x_{n}\right)}{2 \mu f^{\prime}\left(y_{n}\right)+2 \beta f^{\prime}\left(x_{n}\right)}\right)\left(\frac{f\left(z_{n}\right)}{f^{\prime}\left(x_{n}\right)}\right) .
\end{gathered}
$$

\subsection{Soleymani Method}

In [1], Soleymani developed a three-step sixth order of convergence method. Since this method is well known we state the essential expressions used in the method as follows:

$$
\begin{gathered}
y_{n}=x_{n}-\left(\frac{2}{3}\right)\left(\frac{f\left(x_{n}\right)}{f^{\prime}\left(x_{n}\right)}\right), \\
z_{n}=x_{n}-\left(\frac{3 f^{\prime}\left(y_{n}\right)+f^{\prime}\left(x_{n}\right)}{6 f^{\prime}\left(y_{n}\right)-2 f^{\prime}\left(x_{n}\right)}\right)\left(\frac{f\left(x_{n}\right)}{f^{\prime}\left(x_{n}\right)}\right), \\
x_{n+1}=z_{n}-\left(\frac{f\left(z_{n}\right)}{f^{\prime}\left(y_{n}\right)+2 f\left[z_{n}, x_{n}, x_{n}\right]\left(z_{n}-y_{n}\right)}\right) .
\end{gathered}
$$

\section{Application of the New Sixth-Order Jarratt-Type Iterative Methods}

The present sixth-order methods given by (2.6), (2.23), and (2.25) are employed to solve nonlinear equations and compare with the Soleymani, Wang et al. type 1, and type 2 methods 
Table 1: Test functions and their roots.

\begin{tabular}{lr}
\hline Functions & Roots \\
\hline$f_{1}(x)=\exp (x) \sin (x)+\ln \left(1+x^{2}\right)$ & $\alpha=0$ \\
$f_{2}(x)=\left(x^{2}-1\right)^{-1}-1$ & $\alpha=\sqrt{2}$ \\
$f_{3}(x)=(x-2)\left(x^{10}+x+1\right) \exp (-x-1)$ & $\alpha=2$ \\
$f_{4}(x)=(x+1) \exp (\sin (x))-x^{2} \exp (\cos (x))-1$ & $\alpha=0$ \\
$f_{5}(x)=\sin (x)^{2}-x^{2}+1$ & $\alpha=1.40449165 \ldots$ \\
$f_{6}(x)=\exp (-x)-\cos (x)$, & $\alpha=-0.666273126 \ldots$ \\
$f_{7}(x)=\ln \left(x^{2}+x+2\right)-x+1$ & $\alpha=4.15259074 \ldots$ \\
$f_{8}(x)=x^{10}-2 x^{3}-x+1$ & $\alpha=0.591448093 \ldots$ \\
$f_{9}(x)=\cos (x)^{2}-5^{-1} x$ & $\alpha=1.08598268 \ldots$ \\
$f_{10}(x)=\sin (x)-2^{-1} x$ & $\alpha=0$ \\
&
\end{tabular}

Table 2: Comparison of various three-point iterative methods.

\begin{tabular}{lcccccc}
\hline Functions & $(2.6)$ & $(2.23)$ & $(2.25)$ & $(3.1)$ & $(3.2)$ & $(3.3)$ \\
\hline$f_{1}(x), x_{0}=0.25$ & $0.440 e-166$ & $0.409 e-185$ & $0.123 e-161$ & $0.284 e-149$ & $0.102 e-144$ & $0.126 e-217$ \\
$f_{2}(x), x_{0}=1.1$ & $0.181 e-78$ & $0.775 e-37$ & $0.146 e-53$ & $0.195 e-41$ & $0.238 e-39$ & $0.174 e-38$ \\
$f_{3}(x), x_{0}=2.1$ & $0.138 e-118$ & $0.126 e-113$ & $0.120 e-112$ & $0.461 e-100$ & $0.533 e-96$ & $0.236 e-118$ \\
$f_{4}(x), x_{0}=-0.5$ & $0.508 e-199$ & $0.104 e-179$ & $0.391 e-202$ & $0.155 e-183$ & $0.745 e-171$ & $0.471 e-187$ \\
$f_{5}(x), x_{0}=0.25$ & $0.708 e-122$ & $0.440 e-80$ & $0.748 e-120$ & $0.199 e-63$ & $0.185 e-50$ & $0.530 e-128$ \\
$f_{6}(x), x_{0}=1.2$ & $0.266 e-183$ & $0.431 e-192$ & $0.416 e-178$ & $0.327 e-164$ & $0.301 e-159$ & $0.131 e-211$ \\
$f_{7}(x), x_{0}=2$ & $0.278 e-193$ & $0.150 e-255$ & $0.285 e-190$ & $0.851 e-176$ & $0.152 e-169$ & $0.528 e-203$ \\
$f_{8}(x), x_{0}=0.25$ & $0.444 e-187$ & $0.343 e-194$ & $0.551 e-186$ & $0.133 e-181$ & $0.153 e-179$ & $0.105 e-192$ \\
$f_{9}(x), x_{0}=1.7$ & $0.941 e-125$ & $0.115 e-164$ & $0.145 e-122$ & $0.805 e-107$ & $0.587 e-100$ & $0.573 e-128$ \\
$f_{10}(x), x_{0}=4.4$ & $0.122 e-157$ & $0.110 e-135$ & $0.231 e-147$ & $0.101 e-129$ & $0.132 e-124$ & $0.559 e-141$ \\
\hline
\end{tabular}

(3.3), (3.2), and (3.1), respectively. To demonstrate the performance of the new sixth-order methods, we use ten particular nonlinear equations. We will determine the consistency and stability of results by examining the convergence of the new Jarratt-type iterative methods. The findings are generalised by illustrating the effectiveness of the sixth-order methods for determining the simple root of a nonlinear equation. Consequently, we will give estimates of the approximate solution produced by the sixth-order methods and list the errors obtained by each of the methods. The numerical computations listed in the tables were performed on an algebraic system called Maple. In fact, the errors displayed are of absolute value, and insignificant approximations by the various methods have been omitted in Tables 1, 2, and 3.

The new three-step Jarratt-type methods require four function evaluations and have the order of convergence six. To determine the efficiency index of these new Jarratt-type methods, we will use Definition 2.2. Hence, the efficiency index of the sixth-order Jarratt-type methods given is $\sqrt[4]{6} \approx 1.565$, which is identical to other established three-point Jarratt-type methods. The test functions and their exact root $\alpha$ are displayed in Table 1 . The difference between the root $\alpha$ and the approximation $x_{n}$ for test functions with initial approximation $x_{0}$, based on $\beta=1$, are displayed in Table 2 . In fact, $x_{n}$ is calculated by using the same total number of function evaluations (TNFEs) for all methods. In the calculations, 12 TNFE are used by each method. Furthermore, the computational order of convergence (COC) is displayed in Table 3. 
Table 3: COC of various iterative methods.

\begin{tabular}{lllllll}
\hline Functions & $(2.6)$ & $(2.23)$ & $(2.25)$ & $(3.1)$ & $(3.2)$ & $(3.3)$ \\
\hline$f_{1}(x), x_{0}=0.25$ & 6.000 & 6.000 & 6.000 & 6.000 & 6.000 & 6.000 \\
$f_{2}(x), x_{0}=1.1$ & 6.010 & 5.622 & 6.010 & 6.001 & 5.997 & 5.665 \\
$f_{3}(x), x_{0}=2.1$ & 6.000 & 6.000 & 6.000 & 6.000 & 6.000 & 5.012 \\
$f_{4}(x), x_{0}=-0.5$ & 6.000 & 5.982 & 6.000 & 5.983 & 5.980 & 5.984 \\
$f_{5}(x), x_{0}=0.25$ & 5.962 & 6.002 & 5.960 & 5.996 & 5.988 & 5.965 \\
$f_{6}(x), x_{0}=1.2$ & 6.000 & 6.000 & 6.000 & 6.000 & 6.000 & 6.000 \\
$f_{7}(x), x_{0}=2$ & 6.000 & 5.991 & 6.000 & 6.000 & 6.000 & 6.000 \\
$f_{8}(x), x_{0}=0.25$ & 6.000 & 5.985 & 6.000 & 6.000 & 6.000 & 6.000 \\
$f_{9}(x), x_{0}=1.7$ & 6.000 & 5.979 & 6.000 & 5.999 & 5.998 & 6.000 \\
$f_{10}(x), x_{0}=4.4$ & 6.000 & 6.000 & 6.000 & 6.000 & 6.000 & 6.000 \\
\hline
\end{tabular}

\section{Remarks and Conclusions}

In this study, we have constructed new sixth-order Jarratt-type methods for solving nonlinear equations. Convergence analysis proves that these new Jarratt-type methods preserve their order of convergence. We have examined the effectiveness of the new Jarratt-type methods by showing the accuracy of the simple root of a nonlinear equation. The main purpose of demonstrating the new sixth-order Jarratt-type methods for many different types of nonlinear equations was purely to illustrate the accuracy of the approximate solution, the stability of the convergence, the consistency of the results, and to determine the efficiency of the new iterative methods. We conjecture that many other variants of Jarratt-type methods may be constructed in a similar form to that presented in this paper. Finally, we conclude that the new three-point methods may be considered a very good alternative to the classical methods.

\section{References}

[1] F. Soleymani, "Revisit of Jarratt method for solving nonlinear equations," Numerical Algorithms, vol. 57, no. 3, pp. 377-388, 2011.

[2] X. Wang, J. Kou, and Y. Li, "A variant of Jarratt method with sixth-order convergence," Applied Mathematics and Computation, vol. 204, no. 1, pp. 14-19, 2008.

[3] X. Wang, J. Kou, and Y. Li, "Modified Jarratt method with sixth-order convergence," Applied Mathematics Letters, vol. 22, no. 12, pp. 1798-1802, 2009.

[4] W. Gautschi, Numerical Analysis: An Introduction, Birkhäuser, Boston, Mass, USA, 1997.

[5] J. F. Traub, Iterative Methods For Solution of Equations, Chelsea Publishing Company, New York, NY, USA, 1977.

[6] S. Weerakoon and T. G. I. Fernando, "A variant of Newton's method with accelerated third-order convergence," Applied Mathematics Letters, vol. 13, no. 8, pp. 87-93, 2000.

[7] P. Jarratt, "Some efficient fourth order multipoint methods for solving equations," BIT, vol. 9, pp. 119$124,1969$. 


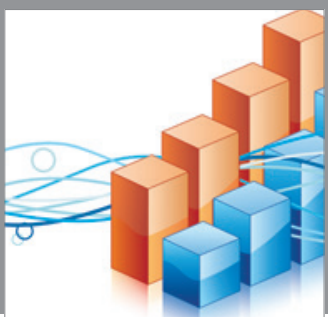

Advances in

Operations Research

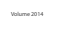

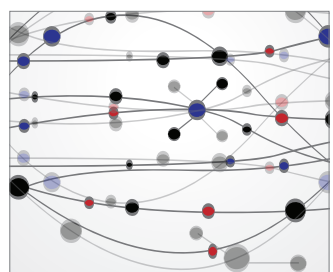

\section{The Scientific} World Journal
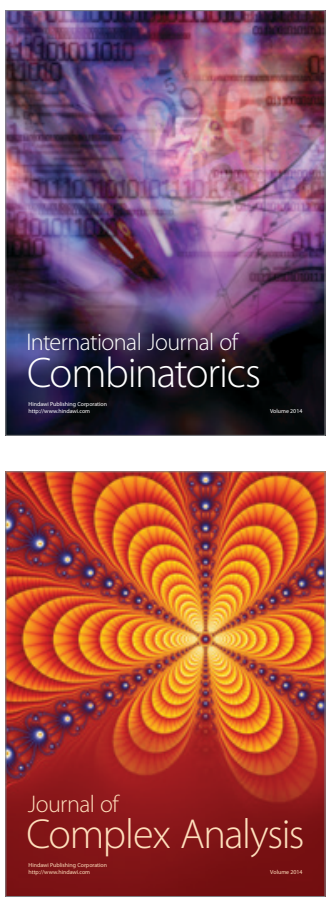

International Journal of

Mathematics and

Mathematical

Sciences
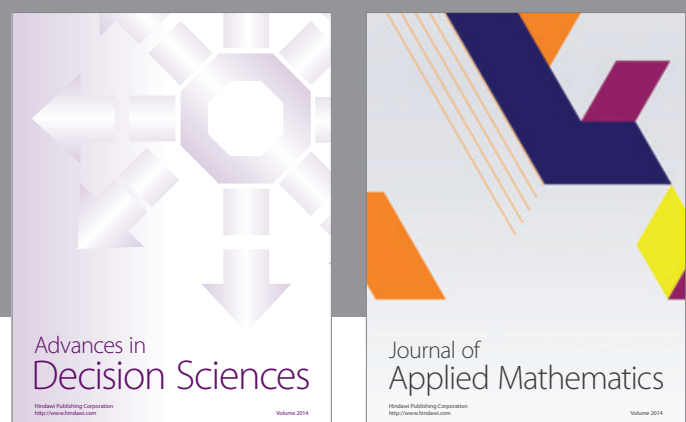

Journal of

Applied Mathematics
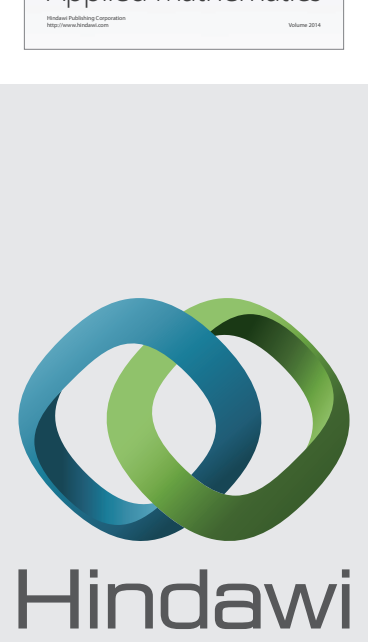

Submit your manuscripts at http://www.hindawi.com
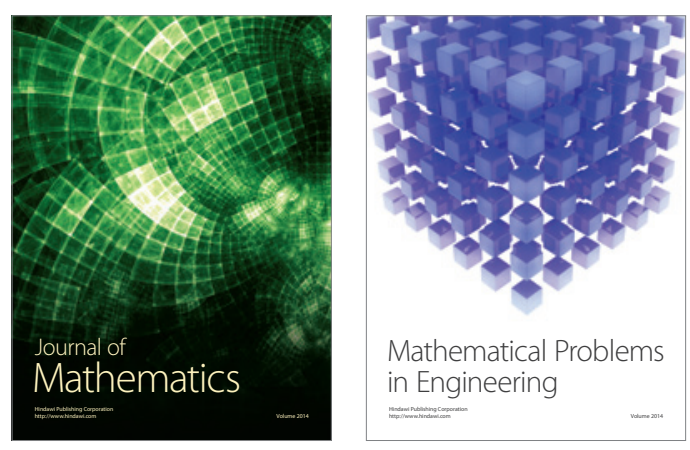

Mathematical Problems in Engineering
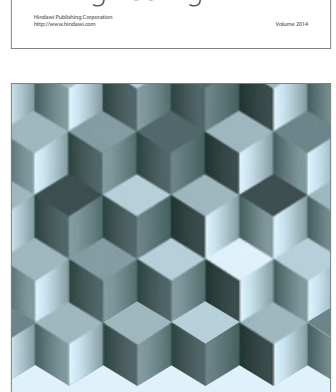

Journal of

Function Spaces
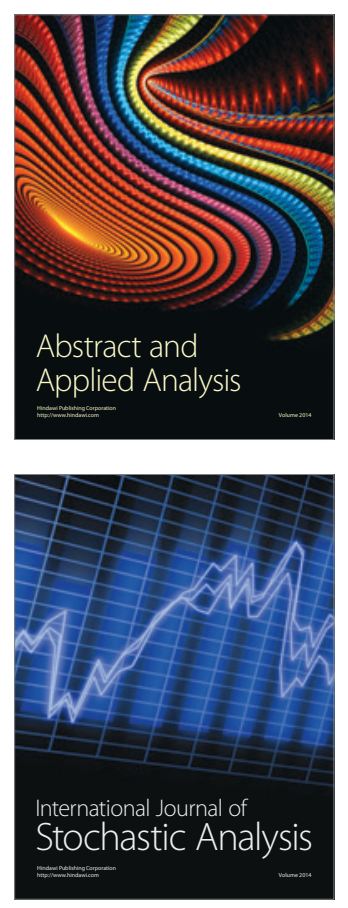

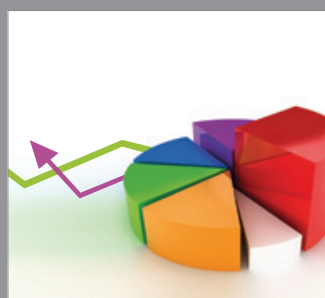

ournal of

Probability and Statistics

Promensencen
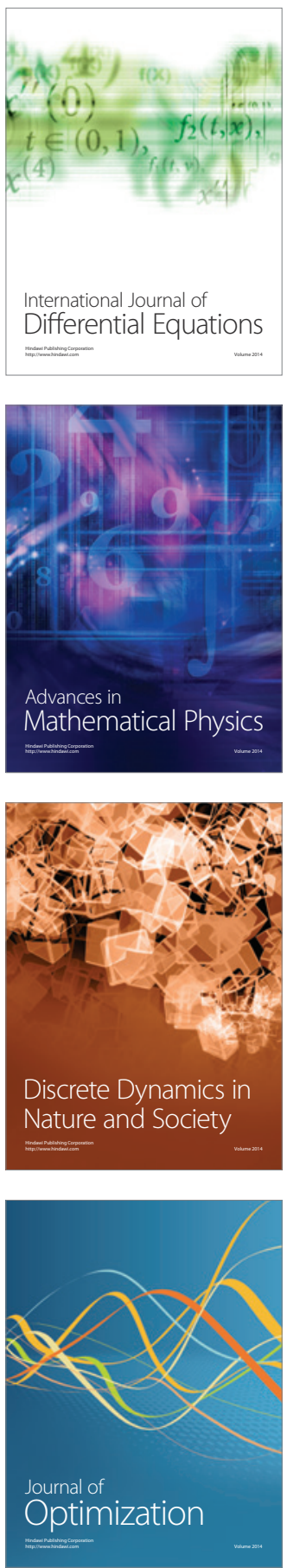